\title{
Modelo de Objetos do openEHR: uma Revisão Sistemática da Literatura e sua relação com métricas de software
}

\section{OpenEHR Object Model: a Systematic Literature Review and its relation to software metrics}

\section{El modelo de objetos openEHR: una sistemática revisión de la literatura y su relación con las métricas de software}

\author{
Reinaldo Araújo de Alkimim | reinaldoalkimim@hotmail.com \\ Universidade FUMEC, Faculdade de Ciências Empresariais, Programa de Pós-Graduação em Sistemas de Informação e \\ Gestão do Conhecimento. Belo Horizonte, MG, Brasil.
}

Fernando Silva Parreiras | fernando.parreiras@fumec.br

Universidade FUMEC, Faculdade de Ciências Empresariais, Programa de Pós-Graduação em Sistemas de Informação e Gestão do Conhecimento. Belo Horizonte, MG, Brasil.

Marcelo Rodrigues dos Santos | marrsantosufmg@gmail.com

Universidade Federal de Minas Gerais, Faculdade de Medicina. Belo Horizonte, MG, Brasil.

Zilma Silveira Nogueira Reis | zilma.medicina@gmail.com

Universidade Federal de Minas Gerais, Faculdade de Medicina, Departamento de Ginecologia e Obstetrícia. Belo Horizonte, MG, Brasil.

Cristiana Fernandes de Muylder | cristiana.muylder@fumec.br

Universidade FUMEC, Faculdade de Ciências Empresariais, Programa de Pós-Graduação em Sistemas de Informação e Gestão do Conhecimento. Belo Horizonte, MG, Brasil.

\section{Resumo}

Dos principais padrões em sistemas de Registro Eletrônico de Saúde (RES), destaca-se a abordagem da Fundação openEHR. Este trabalho apresenta uma Revisão Sistemática da Literatura dos estudos que utilizam esta abordagem com ênfase na utilização do seu Modelo de Objetos. A pesquisa foi realizada nas bases de dados internacionais com base em quatro questões de pesquisa e critérios de inclusão e exclusão definidos. Entre os resultados obtidos, foi possível observar que o continente europeu é o maior centro dos estudos relacionados com a abordagem openEHR, com exceção da Austrália na Oceania. Pode-se concluir que uma versão estável da especificação openEHR contribuiu para o aumento de estudos a partir de 2008. Em relação às métricas de software aplicadas ao modelo do openEHR, até a realização deste trabalho, não se observaram estudos dessa natureza. Além disso, esta revisão possibilitou relacionar as ferramentas para coleta de métricas disponíveis na literatura.

Palavras-chave: Registros eletrônicos de saúde; Desenho de programas de computador; Informática médica; openEHR; Métricas de software. 


\begin{abstract}
In Major standards in EHR systems, highlights the approach of the openEHR Foundation. This paper presents a Systematic Literature Review studies of openEHR's approach with focus on the Object Model. The survey was conducted in international databases based on four research questions and inclusion and exclusion criteria. Between the results obtained, it was observed that the European continent is the largest center of studies related to the openEHR approach, with the exception of Australia in Oceania. May concluded that a stable version of the openEHR specification contributed to the increase of studies from 2008. In relation to software metrics applied to the openEHR model, until the present work, there were no studies. Moreover, this review related the tools to collect metrics available in the literature.
\end{abstract}

Keywords: Electronic health records; Software design; Medical informatics; OpenEHR; Software metrics.

\title{
Resumen
}

De las principales normas en sistemas de Registro Electrónico de Salud (RES), se percibe mejor el enfoque de la Fundación openEHR. Em este ensayo se presenta una revisión sistemática de la Literatura de los estudios que utilizan este enfoque con énfasis en la utilización de su Modelo de Objetos. La encuesta se llevó a cabo en las bases de datos internacionales basado en cuatro preguntas de investigación y criterios de inclusión y exclusión definidos. Entre los resultados, se observó que el continente europeo es el mayor centro de estudios relacionados con el enfoque openEHR, con la excepción de Australia en Oceanía. Es posible llegar a la conclusión que una versión estable de la especificación openEHR contribuyó al aumento de los estudios a partir de 2008. A lo que toca a las métricas de software aplicadas al modelo openEHR, hasta la ejecución del presente ensayo, no existían tales estudios. Además, esta revisión ha permitido relacionar las herramientas para colectar métricas disponibles en la literatura.

Palabras clave: Registros electrónicos de salud; Diseño de programas informáticos; Informática médica; OpenEHR; Métricas de software.

\section{Contribuição dos autores:}

Concepção e desenho do estudo: Reinaldo Araújo de Alkimim, Fernando Silva Parreiras e Marcelo Rodrigues dos Santos. Aquisição, análise ou interpretação dos dados: Reinaldo Araújo de Alkimim, Fernando Silva Parreiras e Marcelo Rodrigues dos Santos. Redação do manuscrito: Reinaldo Araújo de Alkimim.

Revisão crítica do conteúdo intelectual: Zilma Silveira Nogueira Reis e Cristiana Fernandes de Muylder.

Declaração de conflito de interesses: Não há.

Fontes de financiamentos: A pesquisa foi realizada com financiamento parcial da Fundação de Amparo à Pesquisa de Minas Gerais (Fapemig), Edital PPSUS, e Universidade FUMEC.

Considerações éticas: Trata-se de uma revisão de literatura com base em artigos de bases bibliográficas.

Agradecimento/Contribuições adicionais: Não existem.

Histórico do artigo: Submetido: 14.fev.2016 | Aceito: 10.mar.2016 | Publicado: 30.jun.2016

Apresentação anterior: Alkimim, R. A. Modelo de objetos do openEHR: uma avaliação em termos de qualidade de projeto orientado a objeto. 2014. 101 f. Dissertação (Mestrado Profissional em Sistemas de Informação e Gestão do Conhecimento) Universidade FUMEC, Belo Horizonte, 2014. Disponível em: http://www.fumec.br/revistas/sigc/article/view/2270

Licença CC BY-NC atribuição não comercial. Com essa licença é permitido acessar, baixar (download), copiar, imprimir, compartilhar, reutilizar e distribuir os artigos, desde que para uso não comercial e com a citação da fonte, conferindo os devidos créditos de autoria e menção à Reciis. Nesses casos, nenhuma permissão é necessária por parte dos autores ou dos editores. 


\section{Introdução}

Como consequência do crescimento da utilização da Tecnologia da Informação e Comunicação em Saúde e com o acesso compartilhado de informações em nível mundial, tanto os pacientes como os profissionais de saúde esperam que dados clínicos possam ser transmitidos de uma forma segura, confiável e eficiente em todas as unidades de assistência médica. Assim, o histórico integrado de saúde de um paciente pode ser visualizado por prestadores de assistência médica, independentemente do lugar e do momento ${ }^{1}$ Nessa linha de raciocínio, Costa, Tortosa e Maldonado² enfatizam que sistemas de informação das instituições de saúde deveriam ser capazes de se comunicar entre si para apoiar a atenção ao paciente em diversos níveis.

Uma forma de viabilizar estas necessidades é por meio do Registro Eletrônico de Saúde (RES), que pode ser entendido como um repositório de dados do histórico integrado de saúde de um paciente em uma forma processável eletronicamente, armazenado e transmitido com segurança, e acessível a múltiplos usuários ou várias instituições de saúde, que, neste último caso, descreve um RES compartilhável3. Para que isso seja possível, é necessário que exista uma interoperabilidade entre os sistemas de informação para permitir o compartilhamento do registro de saúde, preservando fielmente o significado clínico do profissional de saúde que exerce o papel de autor ${ }^{4}$.

Na busca dessa interoperabilidade, a abordagem que separa o domínio do conhecimento do domínio da informação é apresentada como solução para o RES. Esta abordagem é chamada de modelagem de dois níveis ou multinível. Separar em dois níveis informação e conhecimento facilita a criação e manutenção desses sistemas de informação. Nessa abordagem, é estabelecido um modelo de informação, também denominado modelo de referência, que é usado para representar as propriedades genéricas da informação, e um modelo de conhecimento representado pelo conceito de arquétipos 5 . Dos principais padrões que tratam a interoperabilidade em sistemas RES, destacam-se a Fundação openEHR, CEN 13606, Heath Level Seven (HL7) e o Integrating the Healthcare Enterprise (IHE), DICOM e Medical Markup Language (MML) ${ }^{4,6}$.

Entre os padrões apresentados anteriormente, Lisa Thurston ${ }^{7}$ destaca a representatividade da abordagem da Fundação openEHR citando que ela tem ganhado atenção na comunidade de informática em saúde por fornecer uma solução flexível e extensível que tira proveito da falta de confiança em modelos de dados específicos. Já Linda Velte ${ }^{6}$ cita que a abordagem openEHR é completa, pois possui uma especificação que descreve a gestão, armazenamento, recuperação e troca de dados em sistemas RES. A própria Fundação openEHR credita o sucesso devido à aceitação formal do CEN 13606 como um padrão da Europa e da ISO $^{8}$. Outra vantagem é a existência de um ferramental pronto para uso como Archetype Editor, Archetype Workbench, Template Designer, Clinical Knowledge ${ }^{9}$. Portanto, justifica-se a escolha do padrão openEHR como objeto de estudo deste trabalho.

Entretanto, implementar a especificação do padrão openEHR pode ser uma tarefa trabalhosa. Segundo Velte ${ }^{6}$, trata-se de um padrão complexo e difícil de entender para alguém novo na área. Além disso, a digitalização de registros de saúde é relativamente recente e poucos sistemas de RES têm sido implementados no mundo7 . Em função disso, as instituições de saúde terão de enfrentar muitos desafios durante a implementação de sistemas de RES7 .

Uma forma de minimizar estes desafios é a utilização de métricas de software da área de Engenharia de Software na busca de soluções para os problemas relacionados ao desenvolvimento de software. Métrica de software é o processo que mapeia a característica particular de uma entidade em mundo real, de maneira que seja possível descrevê-la de acordo com as regras definidas e medida em um valor numérico ${ }^{10,11}$. Entre os benefícios das métricas de software, destaca-se a maneira quantitativa de avaliar a qualidade de atributos internos do produto. Isso permite que o engenheiro de software possa avaliar a qualidade do produto antes de ser construído ${ }^{12}$. De acordo com Peters e Pedrycz ${ }^{13}$, são conhecimentos obtidos pela medição de software: custo que afeta o planejamento de projetos futuros; testabilidade e manutenbilidade de processos 
e produtos; eficácia do produto de software; problemas a serem identificados; qualidade do produto; e funcionalidade e facilidade de utilização do produto.

Levando-se em conta os benefícios e conhecimentos que podem ser adquiridos com a medição de software, é justificada a sua utilização em sistemas de RES para minimizar os desafios que as instituições de saúde terão que enfrentar, conforme citado anteriormente. Especificamente, as métricas de Orientação a Objetos (OO) são as mais direcionadas ao padrão openEHR, visto que ele possui um Modelo de Objetos que utiliza os conceitos de OO.

A abordagem OO pode melhorar o desenvolvimento de software, incluindo fatores como maior reusabilidade, que é a medida que um programa ou parte dele pode ser reutilizado em outro aplicativo, e extensibilidade, que é uma característica de adicionar novos aspectos ao software com pouco impacto ${ }^{12,14}$. Com o intuito de ajudar os gerentes e desenvolvedores a atingir esses objetivos, uma variedade de métricas de $\mathrm{OO}$ tem sido proposta para ajudar a controlar projetos de software ${ }^{15}$. Essas métricas podem auxiliar também na manutenção de software para prever esforço de manutenção.

Diante deste cenário, o objetivo deste trabalho éidentificar os estudos que utilizam a abordagem da fundação openEHR e quais fazem uso de métricas de software de forma colaborativa. Especificamente, pretende-se responder às seguintes questões de pesquisa: qual a distribuição geográfica dos estudos do openEHR? (Q1); como foi a evolução anual dos estudos do openEHR desde a primeira especificação em 2004? (Q2); nos estudos encontrados, quantos envolvem métricas de software? (Q3); e quais as ferramentas disponíveis para coleta de métricas de OO que podem ser utilizadas no Modelo de Objetos do openEHR? (Q4).

\section{Metodologia}

Utilizou-se o método de Revisão Sistemática da Literatura ou Systematic Literature Review (SLR) por meio de identificação e interpretação das pesquisas disponíveis na literatura por tratar-se de um método robusto para mapear onde existe pouca pesquisa relevante, ou mesmo evidenciar que nenhuma pesquisa tenha sido feita. Assim, tem sido utilizado como um instrumento de pesquisa comum na Engenharia de Software empírica ${ }^{16,17}$.

A pesquisa foi realizada nas seguintes bases de dados internacionais: ACM Digital Library ${ }^{18}$, Academic OneFile $^{19}$, Biblioteca Digital de Teses e Dissertações (BDTD) ${ }^{20}$, Biblioteca Virtual em Saúde (MEDLINE e LILACS) ${ }^{21}$, BioMed Central ${ }^{22}$, Cambridge Journals ${ }^{23}$, Engineering Village ${ }^{24}$, Crossref ${ }^{25}$, Directory of Open AccesS Journals ${ }^{26}$, EBSCO(27), Emerald ${ }^{28}$, IEEE Xplore ${ }^{29}$, Inspec ${ }^{30}$, ISI Web of Science ${ }^{31}$, SAGE Journals ${ }^{32}$, ScienceDirect ${ }^{33}$, Scopus ${ }^{34}$, Springer ${ }^{35}$, U.S. National Library of Medicine ${ }^{36}$ e Wiley ${ }^{37}$. 
Foram definidas duas estratégias de pesquisa. Uma para as questões de Q1 a Q3, que é exibida na Figura 1, e outra para questão de pesquisa Q4, que é exibida na Figura 2.

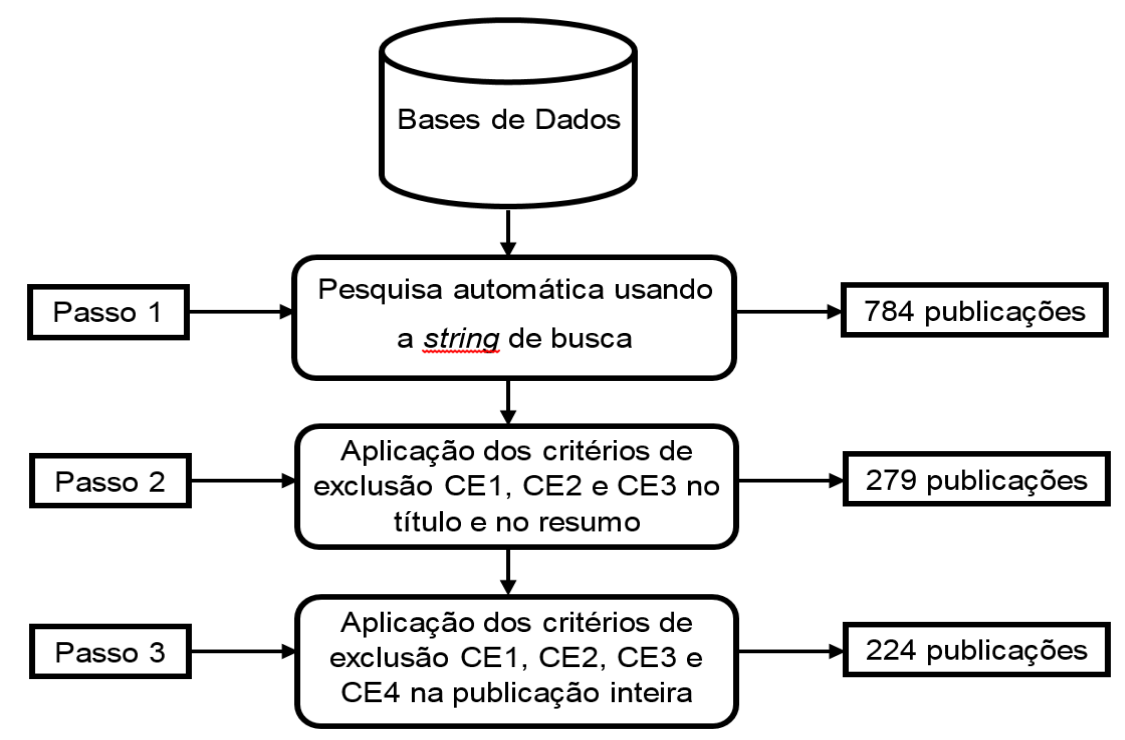

Figura 1. Estratégia de pesquisa para as questões de pesquisa Q1 a Q3 Fonte: Elaboração dos autores.

Para as questões de pesquisa Q1 a Q3 foi utilizada uma string de busca a partir das palavras-chave em inglês Registro Eletrônico de Saúde e openEHR para retornar todos os estudos de RES que envolvam a fundação openEHR. Foi necessário utilizar duas variações da palavra-chave Registros Eletrônicos de Saúde, pois foi identificada a existência de estudos que utilizam estas variações em inglês. Assim, a string de busca ficou da seguinte forma: (Electronic Health Record and openEHR) OR (Electronic Medical Record and openEHR) OR (Electronic Medical System and openEHR). Para a questão de pesquisa Q4 foi utilizado uma string de busca a partir das seguintes palavras-chave em inglês: Ferramenta e Métrica e Orientação a Objeto. Assim, a string de busca ficou da seguinte forma: (Metric AND Tool AND Object-Oriented) OR (Metric AND Tool AND OO). 
Como seleção, foram considerados os critérios de inclusão:

CI1) idiomas português, inglês e espanhol;

CI2) artigos completos publicados em periódicos e conferências; e

$\mathrm{CI} 3$ ) teses e dissertações defendidas.

Como critérios de exclusão:

CE1) ensaios;

CE2) artigos de revisão;

CE3) artigos em duplicidade;

CE4) trabalhos em que a abordagem openEHR existe apenas nas referências, não sendo citada no estudo para as questões de pesquisa Q1 a Q3; e CE5) ferramentas que não estão disponíveis para download para questão de pesquisa Q4.

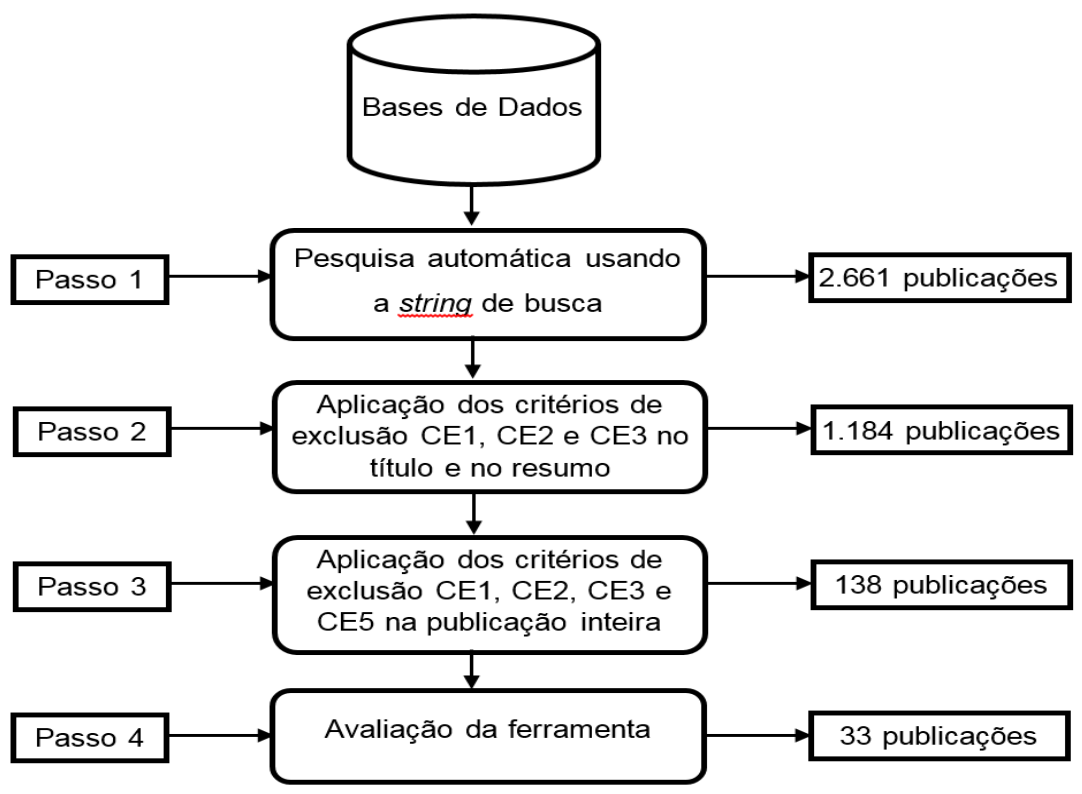

Figura 2. Estratégia de pesquisa para a questão de pesquisa Q4 Fonte: Elaboração dos autores.

Os resultados obtidos são discutidos na próxima seção.

\section{Resultados e discussão}

Os resultados foram divididos de acordo com as questões de pesquisa Q1 a Q4, considerando que nas questões de pesquisa Q1 a Q3 foram obtidos 224 estudos, e na questão de pesquisa Q4 foram obtidos 33 estudos, de acordo com suas respectivas estratégias de pesquisa.

Para a questão de pesquisa Q1, os projetos estão distribuídos geograficamente por 38 países.

Estudos colaborativos entre países foram contabilizados em todos os países envolvidos, exceto quando era aplicado ou financiado por um órgão de um país específico. Nesse caso, foi creditado somente o país de aplicação. 
A Figura 3 exibe o resultado dessa questão de pesquisa em números de publicações.

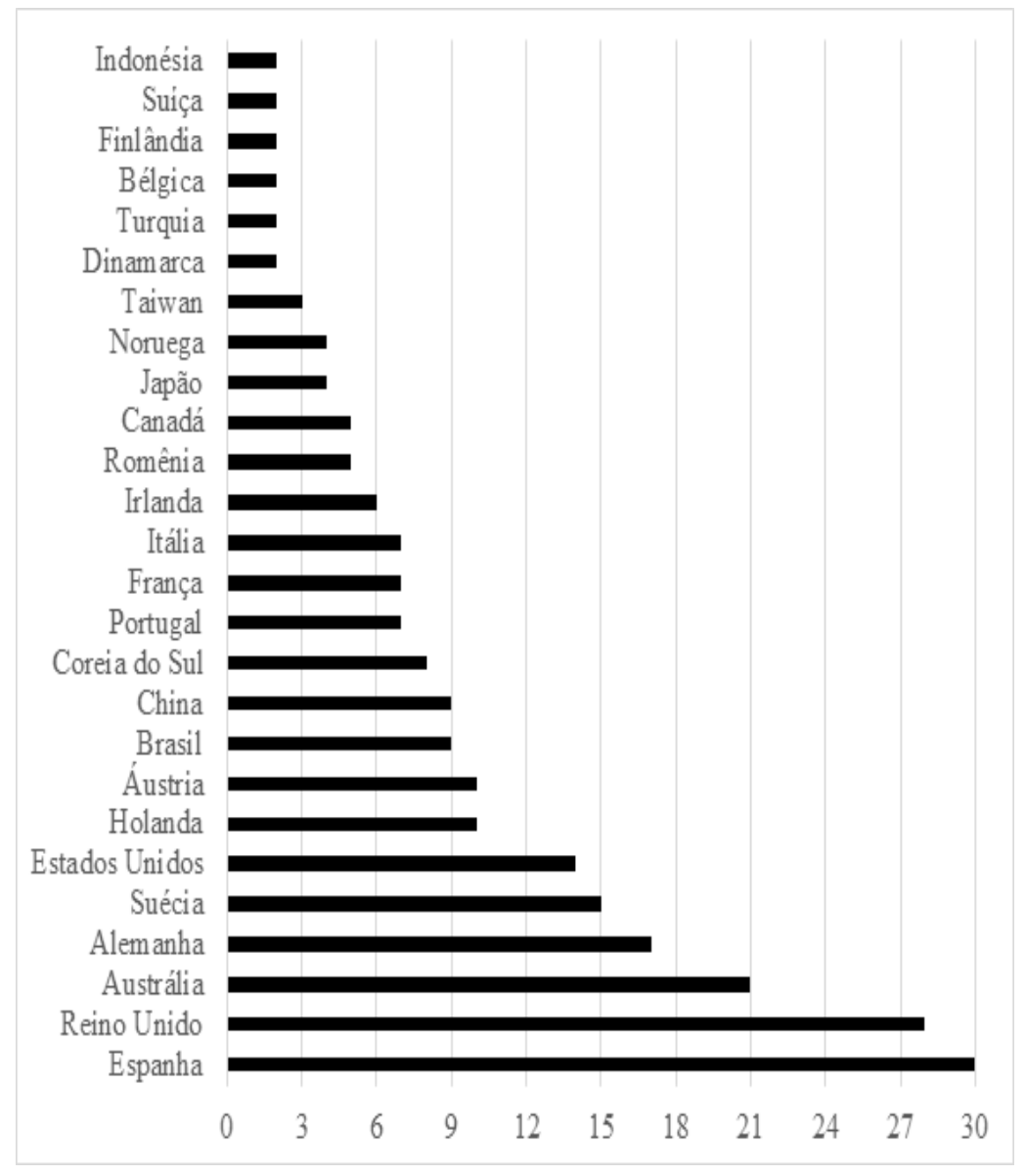

Figura 3. Distribuição geográfica dos estudos relacionados ao openEHR Fonte: Elaboração dos autores.

Nesse resultado não são apresentados os países com apenas um estudo encontrado. São eles: Eslovênia, Nova Zelândia, Hungria, Uruguai, Israel, República Tcheca, Peru, Tunísia, Colômbia, Grécia e Índia.

Dentre os países de destaque, com relação ao Reino Unido e à Austrália, era esperada uma quantidade de estudos significativa, visto que a Fundação openEHR foi fundada pela University College London do Reino Unido e pela Ocean Informatics Pty Ltd da Austrália ${ }^{38}$. Além disso, a maior parte dos conselheiros da Fundação openEHR são professores-doutores desses dois países, o que ajuda a disseminar os estudos.

A Espanha foi o país com a maior quantidade de estudos encontrados, o que se deve, principalmente, aos excelentes estudos que são reconhecidos pela Fundação openEHR ${ }^{39}$ : desenvolvimento de bibliotecas Java para traduzir arquétipos openEHR para OWL ${ }^{40}$; editor arquétipo de referência chamado LinkEHR ${ }^{41}$; projeto POSEACLE para facilitar a gestão semântica de informação e conhecimento em RES ${ }^{42-44 " ; ~ p r o j e t o ~}$ gestão de terminologias para arquétipos ${ }^{45,46}$.

A Alemanha também tem projetos destacados pela Fundação openEHR: expressão de conjuntos de dados

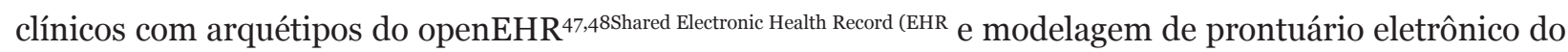
paciente usando a abordagem openEHR ${ }^{49}$.

A Suécia possui uma boa contribuição de estudos devido ao programa de doutorado da Karolinska Institutet, o qual explora a tecnologia RES, em especial a abordagem openEHR. O Brasil foi o único país sul-americano em destaque por contribuição das teses de doutorado concluídas ${ }^{3,50}$ e por linhas de pesquisas existentes em universidades e hospitais ${ }^{51-54}$. 
Em uma análise geral, foi observada a predominância dos estudos no continente europeu, o qual representa $65,29 \%$, seguido do continente asiático com 11,16\%. Os estudos realizados no Brasil compõem praticamente $4,96 \%$ do total do continente sul-americano.

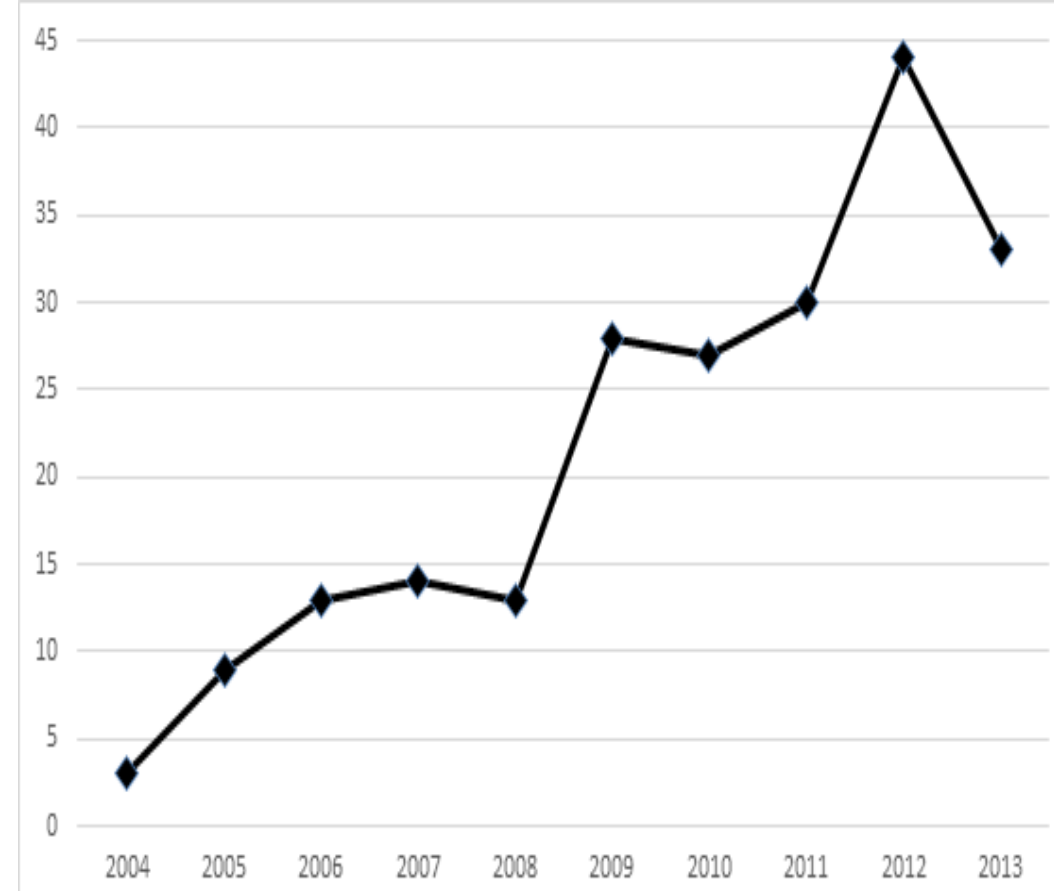

Figura 4. Evolução anual dos estudos relacionados ao openEHR Fonte: Elaboração dos autores.

A questão de pesquisa Q2 analisou a evolução anual dos estudos do openEHR desde a primeira especificação do openEHR em 2004. A Figura 4 exibe o resultado dessa questão de pesquisa.

De 2004 a 2008, a cada ano, a Fundação openEHR liberou uma nova versão da especificação, o que corrobora para a evolução regular que houve nesse intervalo exibida na Figura 4. A versão de 2008 é a última versão estável da especificação, o que culminava com um aumento nos estudos realizados a partir desse marco. No ano de 2013, houve uma queda no número de estudos, provavelmente por haver publicações que ainda não foram catalogadas nas bases de dados pesquisadas.

A questão de pesquisa Q3 procurou estudos que envolvem métricas de software. Durante essa revisão, o único estudo encontrado foi o trabalho de Ahn et al.55, que tinha como objetivo desenvolver métricas de qualidade para avaliar modelos clínicos detalhados. Nesse estudo, foram desenvolvidas métricas de qualidade baseadas no modelo de qualidade ISO 9126. Entretanto, até o presente momento e com a estratégia de pesquisa utilizada, não foi encontrado nenhum estudo que aborda métricas de software para avaliar o modelo de objetos do openEHR. 
Quadro 1. Resultado das ferramentas encontradas para coleta de métricas de 00

\begin{tabular}{|l|l|}
\hline Ferramenta & Usa código fonte em Java \\
\hline Cantata++ & Não \\
\hline CCCC & Não \\
\hline CKJM & Sim \\
\hline Classycle & Sim \\
\hline DependencyFinder & Sim \\
\hline ES2 & Não \\
\hline InCode & Sim \\
\hline InFusion & Sim \\
\hline Iplasma & Sim \\
\hline JBOOMT & Não \\
\hline Jdepend & Sim \\
\hline Jhawk & Sim \\
\hline Metric (Plugin Eclipse) & Sim \\
\hline MetricView & Não \\
\hline OOMeter & Sim \\
\hline QMOOD++ & Não \\
\hline SDMetrics & Não \\
\hline Software Architecture Analysis Tool (SAAT) & Não \\
\hline SourceMonitor & Sim \\
\hline Understand & Sim \\
\hline UnitMetrics & Sim \\
\hline XLSTAT & Não \\
\hline
\end{tabular}

Fonte: Elaboração dos autores.

A questão de pesquisa Q4 identificou as ferramentas disponíveis para coleta de métricas de OO à disposição na literatura. Como resultado, foram encontradas 33 publicações, entre as quais 22 ferramentas estavam disponíveis para avaliação, conforme Quadro 1.

Das ferramentas encontradas, foi feita uma primeira avaliação para identificar quais eram aplicadas em código fonte em Java, visto que os Modelos de Objetos do openEHR são medidos pela implementação de referência em Java, que é reconhecida pela Fundação openEHR. Foram encontradas ferramentas que avaliam apenas modelos em notação UML, como é o caso de MetricView, SDMetrics e Software Architecture Analysis Tool (SAAT). Esse tipo de ferramenta coleta informações obtidas de diversos diagramas, como diagramas de classes, estados, caso de uso, sequência e componentes. Entretanto, métricas de OO que precisam de implementações em seus métodos ficam impossibilitadas de serem aplicadas. Foram encontrados resultados em que as ferramentas eram aplicadas em código-fonte, mas não permitindo o uso de código-fonte em Java, como no caso de QMOOD++, Cantata++ e JBOOMT.

Por fim, as ferramentas que coletam métricas de $\mathrm{OO}$ em código-fonte Java foram instaladas e avaliadas. As ferramentas Classycle, DependencyFinder, InCode, JDepend, OOMeter, SourceMonitor, Understand e XLSTAT possuem poucas métricas implementadas. Já as ferramentas CKJM, InFusion, iPlasma e JHawk possuem um número maior de métricas implementadas. 


\section{Conclusão}

É possível observar que o continente europeu é o maior centro dos estudos relacionados com a abordagem openEHR. A Austrália na Oceania é uma exceção nesse cenário, devido principalmente ao fato de ter uma organização, a Ocean Informatics Pty Ltd, como uma das fundadoras da Fundação openEHR e por ter professores doutores que fazem parte do conselho dela. Além disso, pode-se concluir que uma versão estável da especificação openEHR contribuiu para o aumento de estudos a partir de 2008. Com isto, espera-se que esta pesquisa contribua para traçar um perfil dos estudos que envolvem a abordagem openEHR para a comunidade científica.

Em relação às métricas de software aplicadas ao modelo do openEHR, até a realização deste trabalho, não se observaram estudos dessa natureza. Essa inexistência de estudos é uma motivação para o desenvolvimento de uma pesquisa futura. Além disso, esta revisão possibilitou relacionar as ferramentas para coleta de métricas de OO disponíveis na literatura que podem ser aplicadas ao Modelo de Objetos da fundação openEHR, contribuindo com trabalhos que pretendam utilizar este tipo de ferramenta.

\section{Referências}

1. Hedayat R. Semantic web technologies in the quest for compatible distributed health records [Internet] [Dissertação (Degree of Master Mathematics and Computer Science)]. [Uppsala]: Uppsala University; 2010 [citado 2 abr 2013]. Disponível em: http://uu.diva-portal.org/smash/record.jsf?pid=diva2:310787

2. Costa CM, Tortosa MM, Maldonado JTFB. Semantic Web technologies for managing EHR - related clinical knowledge. 2009 [citado 20 mar 2013];(Semantic Web). Disponível em: http://cdn.intechopen.com/ pdfs/9396/InTech-Semantic_web_technologies_for_managing_ehr_related_clinical_knowledge.pdf

3. Santos MR. Sistema de registro eletrônico de saúde baseado na norma ISO 13606: aplicacões na Secretaria de Estado de Saúde de Minas Gerais [Internet] [Tese (Doutorado em Ciência da Informação)]. [Belo Horizonte]: Escola de Ciência da Informação, Universidade Federal de Minas Gerais; 2011 [citado 30 mar 2013]. Disponível em: http://dspace.lcc.ufmg.br/dspace/bitstream/1843/ECIC8L8HFJ/1/tese eci ufmg marcelo rodrigues dos santos 2011.pdf

4. Kalra D. Electronic health record standards. Yearb Med Inf. 2006;136-44.

5. Santos MR, Bax MP, Pessanha C. Uma leitura ontológica da norma ISO 13606 para o Registro Eletrônico de Saúde. In Porto de Galinhas; 2010 [citado 30 mar 2013]. Disponível em: http://sres.saude.mg.gov.br/ upload/manual/PaperArquetiposeontologias-SESMG.pdf

6. Velte LM. Electronic health record repository based on the openEHR standard [Internet] [Dissertação (Mestrado em Engenharia de Computadores e Telemática)]. [Aveiro]: Universidade de Aveiro; 2011 [citado 2 abr 2013]. Disponível em: http://ria.ua.pt/handle/10773/7479

7. Thurston LM. Flexible and extensible display of archetyped data: The openEHR presentation challenge. In: Proceedings of HIC [Internet]. Adelaide: Health Informatics Society of Australia Ltd; 2006 [citado 20 mar 2013]. p. 28-36. Disponível em: http://my.openehr.org/wiki/download/attachments/2949261/006 Thurston.pdf

8. openEHR. openEHR - Foundation [Internet]. 2013a [citado 4 abr 2013]. Disponível em: http://www. openehr.org/about/foundation

9. Atalag K, Yang HY, Warren J. Assessment of Software Maintainability of openEHR Based Health Information Systems-A Case Study In Endoscopy. Electron J Health Inform. 2011;7(1):e3.

10. Lanza M, Marinescu R. Object-Oriented Metrics in Practice: Using Software Metrics to Characterize, Evaluate, and Improve the Design of Object-Oriented Systems [Internet]. Springer; 2006 [citado 11 jun 2013]. Disponível em: http://books.google.com.br/books?hl=pt-BR\&lr=\&id=gdLb gnaMaa0C\&oi=fnd\&pg=PA1\&dq=Object-Oriented+Metrics+in+Practice+autor:LANZA\&ots=s vMtJEIsQ\&sig=wqqIV07_68oqUDtQOGHo8n1c6uU

11. Fenton NE, Pfleeger SL. Software metrics: a rigorous and practical approach. 2 ed. Boston: PSW Publishing Company; 1998.

12. Pressman RS. Software Engineering. 7o ed. New York: McGraw-Hill; 2010. 
13. Peters JF, Pedrycz W. Engenharia de Software - Teoria e Prática. 1 ed. Rio de Janeiro: Campus; 2001.

14. Garzas J, Piattini M. Object-Oriented Design - knowledge_principles, heuristics, and best practices. London: Idea Group Publishing; 2007. 175 p.

15. Chidamber SR, Darcy DP, Kemerer CF. Managerial use of metrics for object-oriented software: An exploratory analysis. Softw Eng IEEE Trans On. 1998;24(8):629-39.

16. MacDonell S, Shepperd M, Kitchenham B, Mendes E. How reliable are systematic reviews in empirical software engineering? Softw Eng IEEE Trans On. 2010;36(5):676-87.

17. Petticrew M, Roberts $\mathrm{H}$. Systematic reviews in the social sciences: A practical guide [Internet]. Wiley. com; 2008 [citado 5 nov 2013]. Disponível em: http://books.google.com.br/books?hl=pt-BR\&lr=\&id=ZwZ 0C\&oi=fnd\&pg=PR5\&dq=\%22Systematic+Reviews+in+the+Social+Sciences\%22\&ots=wWW0xUNTMw \&sig=sTXvGKnXP0c1q1LUnp7HIMHTNHs

18. ACM Digital Library [Internet]. [citado 23 fev 2013]. Disponível em: http://dl.acm.org/

19. Academic OneFile [Internet]. [citado 25 fev 2013]. Disponível em: http://go.galegroup.com/ auth/capmAuthentication.do?userGroupName=capes2\&origURL=http $\% 3 \mathrm{~A} \% 2 \mathrm{~F} \% 2 \mathrm{Fgo}$.galegroup. com\%2Fps\%2FredirectSearch.AAAAAAdo\%3Fpage\%3DdispBasicSearch.do\%253FprodId\%253DAONE\% 2526userGroupName\%253Dcapes2\%26prodId\%3DAONE\%26userGroupName\%3Dcapes2\%26authCoun t\%3D1\&productShortName=AONE\&productLongName=Academic+OneFile\&isStartUrlRequest=false\&isC ookieCheckDone $=$ false

20. Biblioteca Digital de Teses e Dissertações [Internet]. [citado 14 fev 2013]. Disponível em: http://bdtd. ibict.br/vufind/

21. Biblioteca Virtual de Saúde [Internet]. [citado 5 fev 2013]. Disponível em: http://bvsalud.org/

22. BioMed Central [Internet]. [citado 2 mar 2013]. Disponível em: https://www.biomedcentral.com/

23. Cambridge Journals [Internet]. [citado 7 mar 2013]. Disponível em: http://journals.cambridge.org/ action/login

24. Engineering Village [Internet]. [citado 16 fev 2013]. Disponível em: http://www.engineeringvillage.com/

25. Crossref [Internet]. [citado 6 mar 2013]. Disponível em: http://www.crossref.org/

26. Directory of Open Access Journals [Internet]. [citado 2 mar 2013]. Disponível em: https://doaj.org/

27. EBSCO [Internet]. [citado 28 jan 2013]. Disponível em: http://search.ebscohost.com/

28. Emerald [Internet]. [citado 9 mar 2013]. Disponível em: http://www.emeraldinsight.com/

29. IEEE Xplore [Internet]. [citado 7 fev 2013]. Disponível em: http://ieeexplore.ieee.org/Xplore/home.jsp

30. Inspec [Internet]. [citado 28 fev 2013]. Disponível em: http://inspecdirect-service.theiet.org/private/ home.aspx

31. Web of Science [Internet]. [citado 19 fev 2013]. Disponível em: http://webofknowledge.com/WOS

32. SAGE Journals [Internet]. [citado 2 mar 2013]. Disponível em: http://online.sagepub.com/

33. ScienceDirect [Internet]. [citado 17 fev 2013]. Disponível em: $\underline{\text { http://www.sciencedirect.com/ }}$

34. Scopus [Internet]. [citado 4 fev 2013]. Disponível em: https://www.scopus.com

35. Springer [Internet]. [citado 13 fev 2013]. Disponível em: http://www.springer.com/gp/

36. U.S. National Library of Medicine [Internet]. [citado 27 fev 2013]. Disponível em: https://www.nlm.nih. gov/

37. Wiley [Internet]. [citado 15 fev 2013]. Disponível em: http://onlinelibrary.wiley.com/

38. Kalra D, Beale T, Heard S. The openEHR Foundation. Stud Health Technol Inform. 2005;115:153-73.

39. openEHR. openEHR - Academic Research [Internet]. 2013b [citado 16 out 2013]. Disponível em: http:// www.openehr.org/who is using openehr/academic research

40. Lezcano L, Sicilia M-A, Rodríguez-Solano C. Integrating reasoning and clinical archetypes using OWL ontologies and SWRL rules. J Biomed Inform. abr 2011;44(2):343-53. 
41. Maldonado JA, Moner D, Boscá D, Fernández-Breis JT, Angulo C, Robles M. LinkEHR-Ed: A multireference model archetype editor based on formal semantics. Int J Med Inf. 2009;78(8):559-70.

42. Maldonado JA, Costa CM, Moner D, Menárguez-Tortosa M, Boscá D, Giménez M, et al. Using the ResearchEHR platform to facilitate the practical application of the EHR standards. J Biomed Inform. ago 2012;45(4):746-62.

43. Martínez Costa $C$, Menárguez-Tortosa M, Fernández-Breis JT. Clinical data interoperability based on archetype transformation. J Biomed Inform. out 2011;44(5):869-80.

44. Martínez-Costa C, Menárguez-Tortosa M, Fernández-Breis JT. An approach for the semantic interoperability of ISO EN 13606 and OpenEHR archetypes. J Biomed Inform. Out 2010;43(5):736-46.

45. Allones JL, Taboada M, Martinez D, Lozano R, Sobrido MJ. SNOMED CT module-driven clinical archetype management. J Biomed Inform. jun 2013;46(3):388-400.

46. Meizoso García M, Allones I, Luis J, Martínez Hernández D, Iglesias T, Jesús M. Semantic similarity-based alignment between clinical archetypes and SNOMED CT: An application to observations. Int J Med Inf. ago 2012;81(8):566-78.

47. Duftschmid G, Rinner C, Kohler M, Huebner-Bloder G, Saboor S, Ammenwerth E. The EHR-ARCHE project: Satisfying clinical information needs in a Shared Electronic Health Record System based on IHE XDS and Archetypes. Int J Med Inf. dez 2013;82(12):1195-207.

48. Garde S, Hovenga E, Buck J, Knaup P. Ubiquitous information for ubiquitous computing: expressing clinical data sets with openEHR archetypes. Stud Health Technol Inform. 2006;124:215-20.

49. Buck J, Garde S, Kohl CD, Knaup-Gregori P. Towards a comprehensive electronic patient record to support an innovative individual care concept for premature infants using the openEHR approach. Int J Med Inf. agosto de 2009;78(8):521-31.

50. Dias RDM. Modelagem do padrão TISS por meio do enfoque dual da Fundação openEHR [Internet]. Universidade do Estado do Rio de Janeiro. Faculdade de Ciências Médicas; 2011 [citado 18 out 2013]. Disponível em: http://bases.bireme.br/cgi-bin/wxislind.exe/iah/online/? IsisScript=iah/iah. xis\&src=google\&base $=$ LILACS\&lang=p\&nextAction=lnk\&exprSearch=691823\&indexSearch=ID

51. Alvernaz C, Cook TW, Cavalini LT. Migration of a Pre-hospital Cardiology Emergency System from Data Model to Multilevel Modeling. SIGHIT Rec. março de 2012;2(1):9-9.

52. acelar-Silva GM, Sousa-Santos RF, Cruz-Correia RJ. Creating openEHR content to different moments of care: Obstetrics emergency scenario. In: 2013 IEEE 26th International Symposium on Computer-Based Medical Systems (CBMS). 2013. p. 361-4.

53. Bacelar-Silva GM, Cesar H, Braga P, Guimaraes R. OpenEHR-based pervasive health information system for primary care: First Brazilian experience for public care. In: 2013 IEEE 26th International Symposium on Computer-Based Medical Systems (CBMS). 2013. p. 572-873.

54. Moraes JLC, Souza WL, Pires LF, Cavalini LT, Prado AF. A novel architecture for message exchange in Pervasive Healthcare based on the use of Intelligent Agents. In: 2013 ACS International Conference on Computer Systems and Applications (AICCSA). 2013. p. 1-8.

55. Ahn S, Huff SM, Kim Y, Kalra D. Quality metrics for detailed clinical models. Int J Med Inf [Internet]. 2012 [citado 4 abr 2013]; Disponível em: http://www.sciencedirect.com/science/article/pii/ $\underline{\mathrm{S} 1386505612001906}$ 